\title{
Trotz Kulturunterschied zur richtigen Diagnose
}

\author{
Dieser Ausgabe von MMW liegt wieder „info praxisteam“ bei, das Magazin für Medizinische \\ Fachangestellte. Hauptthema in Heft 1/16 sind die Migranten. Ihre Versorgung sprengt oft den \\ Rahmen des Alltäglichen, allein schon wegen der Sprachbarriere. Eine neue Serie liefert Tipps.
}

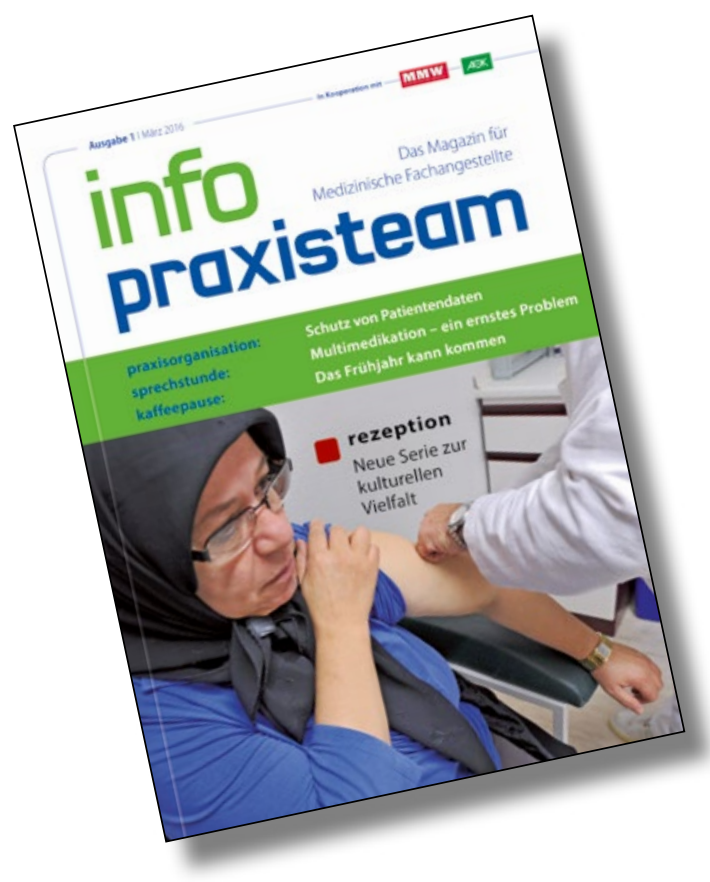

_ Migration ist das politische Thema unserer Zeit, und gerade in den Hausarztpraxen erwachsen daraus ganz praktische Herausforderungen. „info praxisteam" startet dazu in dieser Ausgabe eine neue Serie: „Kulturelle Vielfalt in der Hausarztpraxis“. Im ersten Beitrag geht es um Unterschiede im Rollenverständnis und den Ansichten zur Gesundheit sowie um Sprachbarrieren.

Während in Deutschland die klassische Schulmedizin den Alltag einer Arztpraxis bestimmt, sind in vielen Ländern, aus denen Migranten kommen, magische Praktiken noch weit verbreitet. Wenn Migranten aus Südosteuropa über diffuse Schmerzen klagen, wird mancherorts gerne etwas respektlos ein "Morbus Bosporus“ diagnostiziert. Die kulturellen Hintergründe liefern in vielen Fällen einen Ansatzpunkt für die Therapie. Die Patienten sind manchmal nicht in der Lage, die genaue Lokalisation von Schmerzen zu beschreiben. Sie äußern deshalb z. B. somatische Symptome auf Basis von seelischen Problemen häufig als Bauchschmerzen.

Mancher Patient fühlt sich mit dem Ansinnen, das Unwohlsein genau zu beschreiben, überfordert. Stattdessen ist es nicht ungewöhnlich, dass erwachsene Patienten in der Sprechstunde stöhnen und jammern oder während der Untersuchung laut aufschreien, ohne dass es dafür einen konkreten Anlass gegeben hat. Das starke Stöhnen soll das real erlebte Kranksein („Alles tut weh“) deutlich machen. Oft erwarten die Patienten vom Arzt eine Diagnose ohne eigene Beteiligung.

\section{Neugierige, wertschätzende und akzeptierende Haltung}

Es gibt unter den Migranten einen durchaus relevanten Anteil an Analphabeten und ein großes Wissensdefizit. Die Sprachbarriere kann für Frustration auf beiden Seiten sorgen. Eine neugierige, akzeptierende und wertschätzende Haltung ist deshalb enorm wichtig. Sonst sind hohe Therapieabbruchraten die zwangsläufige Folge. Besser als ein Familienmitglied ist oft eine außenstehende Person als Dolmetscher.

Bei Verständigungsschwierigkeiten mit Patienten können Praxen auf kostenloses Infomaterial zurückgreifen, das unter anderem bei der Bundeszentrale für gesundheitliche Aufklärung (BZgA) bestellt werden kann. Im Angebot sind Therapiepläne als Bildergeschichte oder Anamnesebögen, auf denen Patienten an einer aufgezeichneten Person ihre persönlichen Schmerzpunkte ankreuzen können.

Auch die Krankenkassen bieten fremdsprachiges Informationsmaterial für die Kommunikation mit Migranten an. Von der AOK etwa gibt es den deutsch-türkischen Vorsorgemanager „AOK-Sağlık hizmeti“ als App für Smartphones.

Nun ist es unmöglich, sich auf jede einzelne Kultur einzustellen. Doch das müssen Sie auch nicht. Eine einfache Hilfe zur Kommunikation ist das sogenannte „Business Behaviour"-Modell über soziales Verhalten im beruflichen Alltag. Es wurde von dem US-Amerikaner Richard R. Gesteland entwickelt und lässt sich gut auf die Arztpraxis übertragen. Das Modell wird im Beitrag detaillierter vorgestellt.

In der Rubrik "praxisorganisation“ geht es um den Datenschutz in der Praxis. Dazu gehört sowohl der Schutz gegen Datenklau durch Hacker als auch Vertraulichkeit im täglichen Praxisablauf. Die „sprechstunde“ behandelt das Problem der Polypharmazie.

Dr. Reinhard Merz

\section{„info praxisteam“ im Web}

Unser MFA-Magazin erscheint viermal jährlich. Alle Beiträge sind unter

www.info-praxisteam.de

auch im Internet abrufbar und können direkt kommentiert werden.

Auch für mobile Endgeräte wie Smartphones oder Tablets ist „info praxisteam“ verfügbar. Die App wird sowohl für iOS von Apple als auch für Geräte mit Android-Plattform angeboten. Mehr darüber, wie man die App beziehen kann, erfahren Sie auf der oben angegebenen Website.

Zu den Beiträgen im Heft gibt es von der Redaktion ausgesuchte Verweise auf weiterführende Informationen im Internet. Viele davon sind auch für Sie als Arzt interessant.

Sie erreichen uns per E-Mail unter

redaktion@info-praxisteam.de 